\section{Agios Pharmaceuticals}

\author{
Agios has brought cancer metabolism into vogue and is \\ making hay from tumor cells' well-known hunger for glucose.
}

For a three-year-old company to land a licensing deal worth $\$ 130$ million upfront is surprising, even more so when the assets are preclinical. But in April, the fledgling Agios netted the head-turning deal with Celgene of Summit, New Jersey, based on a biochemical observation about cancer cell metabolism that dates back 85 years. Founders Craig Thompson at the University of Pennsylvania, Tak Mak of the University of Toronto and Lewis Cantley, transduction expert from the Harvard Medical School and Beth Israel Deaconess Medical Center in Boston, set up Agios in 2007 with headquarters in Cambridge, Massachusetts. They rekindled a discovery made by Otto Warburg in 1924 that virtually all malignant cells choose aerobic glycolysis, an inefficient way to burn glucose that yields two net ATP molecules, rather than the usual oxidative phosphorylation that yields 30 . Because tumor cells need to consume more glucose to maintain ATP levels, glucose withdrawal seemed a promising therapeutic route. But despite manifold efforts, blocking glycolysis failed to yield any anticancer agents.

So it is perhaps surprising that, in 2004, Thompson applied for a National Cancer Institute grant to study how rapid uptake of glucose triggers a fundamental reprogramming of metabolism in cancer cells. Mak, a molecular biologist and immunologist, was asked to conduct a site visit to assess the application. Thompson recalls how Mak hated the idea, and returned to his Toronto lab determined to quash the notion. Two years later, however, Mak had become a convert to the cause. What is more, he had generated a significant body of evidence to show that tumor cell metabolism would be an ideal target for a new generation of anticancer agents that would have little impact on healthy cells.

In 2007, Mak and Thompson organized a symposium on the subject at the American Association for Cancer Research National Meeting in Los Angeles. One of the speakers was Lewis Cantley, from Harvard Medical School, who had discovered the phosphoinositide-3 kinase pathway that Thompson believed was an important link between metabolism and malignant transformation. Relaxing at a table after the session, the three scientists began throwing ideas around and before the night was over, had the basics of a company sketched out on a napkin. Taking advantage of a connection that
Mak had to angel investors, the three procured "a couple million dollars" and hired Shin-San Su from the Biomedical Engineering Research Laboratory in Taiwan to run Agios' scientific efforts and help the three scientific founders to develop a business plan.

In 2008, the Agios team used a proteomic screen for phosphotyrosine binding proteins using stable isotope labeling of amino acids (SILAC) of HeLa cell culture lysates followed by flow over phosphotyrosine/unphosphorylated peptide library affinity matrices and analysis by liquid chromatography tandem mass spectrometry (Nature 452, 181-186, 2008). The screen showed, for the first time, a mechanistic link between an enzyme involved in glucose metabolism-the phosphotyrosine-binding pyruvate kinase M2 isozyme (PKM2) — and tumor cell growth. With these early data in hand, Agios headed to the venture well and in July 2008 closed \$33 million in Series A funding. The cash provided by Boston-based Third Rock Ventures, Flagship Ventures in Cambridge, Massachusetts and $\mathrm{ARCH}$ Venture Partners, from Chicago, allowed Agios to move forward quickly.

Last November, Agios researchers also reported an association between a single amino acid substitution in the isocitrate dehydrogenase 1 (IDH1) enzyme and the development of brain cancer (Nature 426, 739-742, 2009). And just a few months later, the company clinched the lucrative deal with Celgene, the latter taking an exclusive option to license any clinical candidates from their discovery and early development work at the end of phase 1 trials. Agios could receive a further $\$ 120$ million in milestones plus royalties on each licensed program, and the option to codevelop and co-market the products.

Agios' platform combines large-scale metabolomic profiling to initially identify enzymes, followed by a genetic approach to search for mutations in the pathway and X-ray crystallography to identify a specific site on the enzyme. In the 2009 Nature paper, they show that three different mutations in arginine 132 of the IDH1 enzyme result in an entirely new function capable of reducing $\alpha$-ketoglutarate to $R(-)-2-$ hydroxyglutarate. Concentrations of the latter molecule increase by a 100 -fold in human brain tumors that contain mutant IDH1 ( 70\% of gliomas and glioblastomas), pointing to a common pathogenic mechanism and the potential

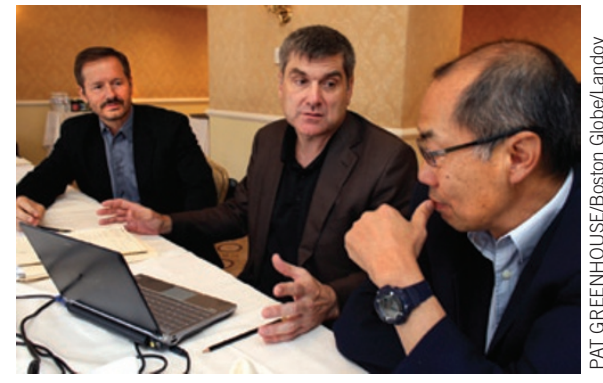

Agios founders (left to right): Lewis Cantley, Craig Thompson and Tak Mak.

of IDH1 as a therapeutic intervention point. Rather than sequence the $I D H 1$ gene to identify mutations, however, Agios plans to screen for metabolites, such as $R(-)$-2-hydroxyglutarate. David Schenkein, who was senior vice president for clinical hematology/oncology at Genentech before joining Agios, says that the company intends to base go/no-go decisions on the availability of biomarker-therapeutic pairs, in a similar manner to the S. San Francisco big biotech, now part of Roche.

Agios is also developing imaging agents to visualize tumors by identifying hotspots of metabolic activity. For instance, the glucose analog 2 - $\left[{ }^{18} \mathrm{~F}\right]$-fluoro-2-deoxy-D-glucose as a radioactive tracer is taken up rapidly but metabolized slowly by cancer cells-the very effect Warburg described. This can be easily quantified using positron emission tomography (PET) to provide a real-time assessment of tumor metabolism.

Targets disclosed thus far by Agios and Celgene are IDH1 and PKM2, but others will be added, says Schenkein. According to Matthew Vander Heiden, who is at the Koch Institute for Integrative Cancer Research, Massachusetts Institute of Technology, in Boston, the firm's biggest challenge will be to stay focused on their strength and resist sliding back into a traditional gene-biotech company. Vander Heiden notes that many researchers are now looking back and realizing that maybe they didn't learn all the biochemistry in the 1940s, and that there is still some hay to be made. Matej Orešič, systems biology and bioinformatics professor at the VTT Technical Research Centre of Finland, believes that despite past failures, with the emergence and maturing of metabolomics, there is a strong case for looking into cancer metabolism. He points out, however, that although IDH1 is a promising case study, it is still very far from the clinic. Given the systemic complexity of metabolism, says Orešič, the case for the Agios therapeutic strategy will be much stronger once demonstrated in a physiological setting. Joe Alper with additional reporting by Lisa Melton 